\title{
Preservation of Geological Information Using a Repository System on Langkawi Geoparks
}

\author{
Aziz Deraman ${ }^{1,2}$, Jamaiah H. Yahaya ${ }^{1,3}$, Juhana Salim ${ }^{1}$, Sufian Idris ${ }^{1}$, Ibrahim \\ Komoo ${ }^{4}$, Mohd. Shafeea Leman ${ }^{4}$ and Tanot Unjah ${ }^{4}$ \\ ${ }^{1}$ Faculty of Information Science and Technology, Universiti Kebangsaan Malaysia, Bangi, Selangor, \\ Malaysia \\ ${ }^{2}$ Universiti Malaysia Terengganu, Kuala Terengganu, Terengganu, Malaysia \\ ${ }^{3}$ College of Arts and Sciences, Universiti Utara Malaysia, Sintok, Kedah, Malaysia \\ ${ }^{4}$ Institute for Environment and Development (LESTARI), Universiti Kebangsaan Malaysia, Bangi , \\ Selangor, Malaysia
}

\begin{abstract}
The development of a repository system is a valuable solution that can assure the preservation of geological information and would provide a more sustainable tool for future references. This research attempted to establish on how geological information could be modeled into various forms of digital object (DO) to enable access and sharing of geological information. The main objective of this research was to provide a sustainable and maintainable repository system that would preserve geological data in an efficient manner. In this effort, the Langkawi Geodiversity data will be captured, modeled and kept in a single repository system. Samples were collected from Langkawi geoparks and analysed in order to understand the complexity of the information needed as well as their relationships. The DO structure and definition were formulated based on the collected samples and tested to a certain level of optimization. With regard to toolset design and implementation, a commercial database engine was used to implement the development of the repository named as MyGeo-RS. The Langkawi geodiversity data was captured digitized and populated into the repository and was published. This paper presents the development of MyGeo-RS which includes the architecture, the design of geodiversity repository and the implementation. The preserved data provided by this system is suitable for tourism and public users and at the same time would also contribute to scientific applications.
\end{abstract}

Keywords: geological information, Langkawi Geoparks, repository system, digital object structure

\section{Introduction}

According to Komoo (2003), all geological and landscape resources have two opposing value sets: extractive and conservative activities. In the conservative concept, a new paradigm earth physical resource is regarded as having value in the context of knowledge, history and culture.
The geoconservation idea arose mainly from the need to protect heritage resources that are already recognized by the general public (or naturalists and geologists) in the context of preserving the landscape beauty for recreation or outstanding heritage value. The philosophy is embodied in the UNESCO's Geopark approach, which provides guidelines for identifying, and

Copyright (C) 2011 Aziz Deraman, Jamaiah H. Yahaya, Juhana Salim, Sufian Idris, Ibrahim Komoo, Mohd. Shafeea Leman and Tanot Unjah.This is an open access article distributed under the Creative Commons Attribution License unported 3.0, which permits unrestricted use, distribution, and reproduction in any medium, provided that original work is properly cited. Contact author: Aziz Deraman E-maill: ad@ftsm.ukm.my 
assessing natural world heritage sites that have outstanding universal value (UNESCO, 1988). A geopark is defined as an area with a geological heritage of significance, with a coherent and strong management structure and where a sustainable economic development strategy is in place (UNESCO, 2008). Geoparks are not just about rocks but they are also concerned with people.

The foundation of the assessment of geological heritage resources is geological diversity or geodiversity. The concept of geodiversity is a new concept amongst geologist that carries a different meaning to different researchers (Komoo, 2003). It refers to the range of geological (bedrock), geomorphological (landform) and soil features, assemblages, systems and processes. The geodiversity concept takes into account the different dimensions shown by a geological exposure or landform. In addition to the variety of characteristic of minerals, rock, fossils, structure and landform features; time, environment and processes are also incorporated in dealing with geodiversity. It contributes to our quality of life in many ways. Its benefits include appreciation, knowledge, products and natural processes (UNESCO, 1988).

Geoheritage value is strongly linked with the specific geodiversity pertaining to significant scientific records for research, education, tourism and industry usability. This diversity includes a varied and complex amount of information and data concerning rock, mineral, fossil, structural and landform diversity that can be found in varied forms and resources.

Similar to the necessity and success that derived biologist to represent and deploy their information digitally into biodiversity information systems, the geologist as well have been attracted to this technology according to the nature of the complexity and diversity of geological datasets and their interrelated components. Therefore, providing theoretical and systematic modeling approaches for organizing and representing those datasets to global access have become one of the arising challenge that required more attention from the researchers and computer professionals in Malaysia. This need has been derived from scientific, educational, research, industrial, economic, social, culture and inspirational values that can be gained. In addition, the lack of awareness from the software professionals to the geodiversity domain remains hidden from the global sharing. Moreover, the preservation of geodiversity datasets is semi or unorganized, as well as the overlook of digital archiving aims is another issue to be considered.

Information system through various research works has been proved to be an appropriate solution to facilitate the organization and archiving of varied and complex information concerned different domains as biodiversity, medical and environment (Aziz, et al., 2005). Therefore, the development of geodiversity information or repository system is believed to be one of the suitable solutions that can assure the management and preservation of geological datasets to be more interesting, accessible and shared to the public at large. Probably, with the advancement of information and communication technology, as well as data mining and knowledge discovery, knowledge about geodiversity can be better represented and explored. This project attempts to establish on how geodiversity components and their relationships can be modeled into various forms of digital object (DO). These sets of digital objects can then be incorporated into primary repository of Geodiversity Repository System (GRS) for easy manipulation of DO's information and knowledge.

In our project, an exclusive geodiversity information system was developed that focused on creating a new digital object model, namely Networked Digital Object (NDO). Our model allows a sharing environment with a knowledge discovery through relating varied and specific DOs among the geodiversity components to produce a new NDO, which represents new information.Together with the input from Langkawi Geopark components and knowledge, GRS can be customized into Langkawi Geopark GRS (LGGRS). With this 
establishment, further research on geological data and expertise and other DO services can be incorporated into the repository. Finally, GRS can be generalized to offer geodiversity knowledge for other places. Bringing GRS technology closer to knowledge based, knowledge management and data mining several models can be proposed for both commercial products and research prototypes.

\section{Literature Review}

For the past decade, organizations, universities and research units from different areas which include fields such as biology, medicine, chemistry and geology have witnessed an exponential growth in digital information available for learning and sharing experiences. There are many collections of digital objects including images, texts, audios, and videos that have great value in a diverse set of fields. As the quantity of information continues to increase and these collections expand, there is a need for a repository system that can provide appropriate storage and access to all these valuable materials in a flexible and extensible manner for the foreseeable future. This need has led many geological organizations and experts to select a geodiversity information system solution. This system can assimilate current collections and accommodate new materials by dealing with highly structured geological data (such as rock, soil, and topographical data) and complex spatial relationships as they become available to global users. In fact, several projects have been developed to handle varied datasets and issues of geological domain based on the developers interest and needs, such as geological hazard spatial information system (Lee and Choi, 2001) and webbased geographic information system (Chang and Park, 2004).

Lee and Choi research work (2001) focused on the datasets that considered the geological hazard management, assessment and prediction in the development of their geological information system. In this work, a geological hazard spatial database (SDB) was designed and constructed. Among the data structures used were coverage (vector data), GRIDs (raster data), and images (raster data). The SDB included geological hazards, basic maps damageable objects, satellite imaging, meteorological data and terrain analysis data. To use the constructed SDBs, a geological hazard spatial information system was developed. In this information system, the SDB output can be selected according to scale. The system used GUI that allowed the development of a user-friendly application. It also featured many functions such as retrieval, identification, edit, and help.

The main objective of Chang and Park study (2004) was to develop a Web-based Geographic Information System (GIS) model for efficient management of borehole and geological data. They claimed that GIS application development adopting internet technology was essential because the efficiency of data usage and knowledge sharing was very important in the developing countries. This project suggested a borehole data standard and corresponding database. More than 10,000 boreholes and other geological data were archived into the database. A prototype for Web-based GIS application was successfully designed to provide systemic interfaces and functions such as geological information search, on-line geological functions, statistical summaries and administrative functions. Geological data from many construction projects should be standardized, structured, archived and properly used through suitable system and applications for efficient management. However, there yet remained many important problems to be solved for advanced GIS applications. One problem came from the requirement of suitable standards for geological data and good design of geological database. Another problem came from the requirement of a suitable modeling approach that was essential to maximize the sharing of geological information and knowledge to solve problems related to geotechnical engineering.

$\mathrm{Xu}$, et al. (1999) developed a query system for China National Biodiversity Information. The objective of this system was to provide metadata to help people to find data and to understand its content and characteristics. The system possessed 
functions such as information search and queries.

Our research focused on geodiversity information in Langkawi Island of Malaysia. Langkawi Island is a well known island with valuable geology sites and rich of geodiversity and natural heritage information. Previously, there were several works on creating geodiversity inventory and individual projects related to Langkawi geoparks. Some of them are Komoo (2003) and Komoo and Leman (1999). Unfortunately, the information created was not integrated and comprehensive because these works were done mostly at the geologists and scattered. Data which was stored in obsolete or inaccessible forms, was poorly standardized and documented. Meanwhile, separated ownership of geology data arose difficulty of information exchange, sharing and comprehensive processing. At the same time, there was no collaboration works done with information technology experts to integrate the geodiversity information, dissemination of the information and monitoring the valuable geodiversity and heritage.

\section{Research Approach}

In this action research, theoretical and experimental approaches were adopted to finally produce a usable repository. Initial study involved collecting sample data of geodiversity for Langkawi. The samples were collected and analysed in order to understand the complexity of the information needed as well as their relationships. The next phase was the data representation formulation. In this phase, a DO structure and definition was formulated and tested to a certain level of optimization (the use of data normalization approach is expected to be beneficial). With a well-defined DO, a workable conceptual repository structure for Langkawi Geodiversity was developed and tested. With regard to toolset design and implementation, a commercial database engine was used to implement the development of the repository named as MyGeo-RS. Once the MyGeo-RS was completed and approved for deployment, the Langkawi geodiversity data was captured, digitized and populated into the repository.

\section{System Analysis}

The target users for MyGeo-RS include tourism sector and the general public who are interested in geodiversity. In the system analysis phase, methods had been undertaken to establish user needs. Initially, the research team consisting of postgraduate students and researchers from two domains; information technology and geology experts held initial discussions on the research domain areas specifically on geodiversity and system design. The discussion revolved around the understanding of the concept of geodiversity from the perspective of geological heritage research, geoconservation and geographical landscape tourism. The research team had visited several geoparks in Langkawi to observe and better understand the information needed as well as their relationships.

The information required to established user needs was decided through a number of focus groups and through several methods including structured interviews, group discussion and workshop and collaboration with Langkawi Development Authorities or best known as LADA. Here, user needs were determined by measure of regular consultations between the development teams led by experienced software engineers and data design with the users who were represented by geology experts through workshops and brainstorming session among these three expert groups. This encouraged a sense of participation in the development process by users. The following phase was the development of architecture.

\section{MyGeo-RS Architecture}

The basic data flow of Langkawi Geodiversity Repository System or MyGeo$\mathrm{RS}$ is represented in an architecture that has been designed and is shown in Figure 1. MyGeo-RS is a metadatabase that will use geodiversity data as the input to produce or present digital objects of the geodiversity domains. Three categories of 
the digital objects representation have been identified and selected which consist of Artifacts Digital Objects, Geosites Digital Objects and Management Objects. The category selection was based on information from our geodiversity experts and was focusing specifically on Langkawi.

The metadatabase consists of three levels of input. The first level contains information on the description of geodiversity types, which we obtain from the geological experts. The second level is the information on description of the data sets that are inter-related from the underlying sources of the geodiversity types described. The third level describes the information on the actual geodiversity data that have been documented and collected in forms of existing databases, files and other digital types, which includes images, reports, maps and video. Geodiversity metadata can then be produced using the data from these three levels of input.

MyGeo-RS accesses the input data through the geodiversity metadata and also the actual geodiversity data, which is processed into the digital objects representation according to the selected categories. One of the modules in MyGeo$\mathrm{RS}$ is the classification module of geodiversity types. At this initial stage, the classification process is categorized into two categories; attributes and services. The attributes are described by our geology experts and in accordance to the geodiversity types agreed. The potential services that have been seen to be of interest are tourism, knowledge and products. The architecture of MyGeo-RS is illustrated in Figure 1. This architecture was originally discussed in our previous paper (Aziz, et al., 2009).

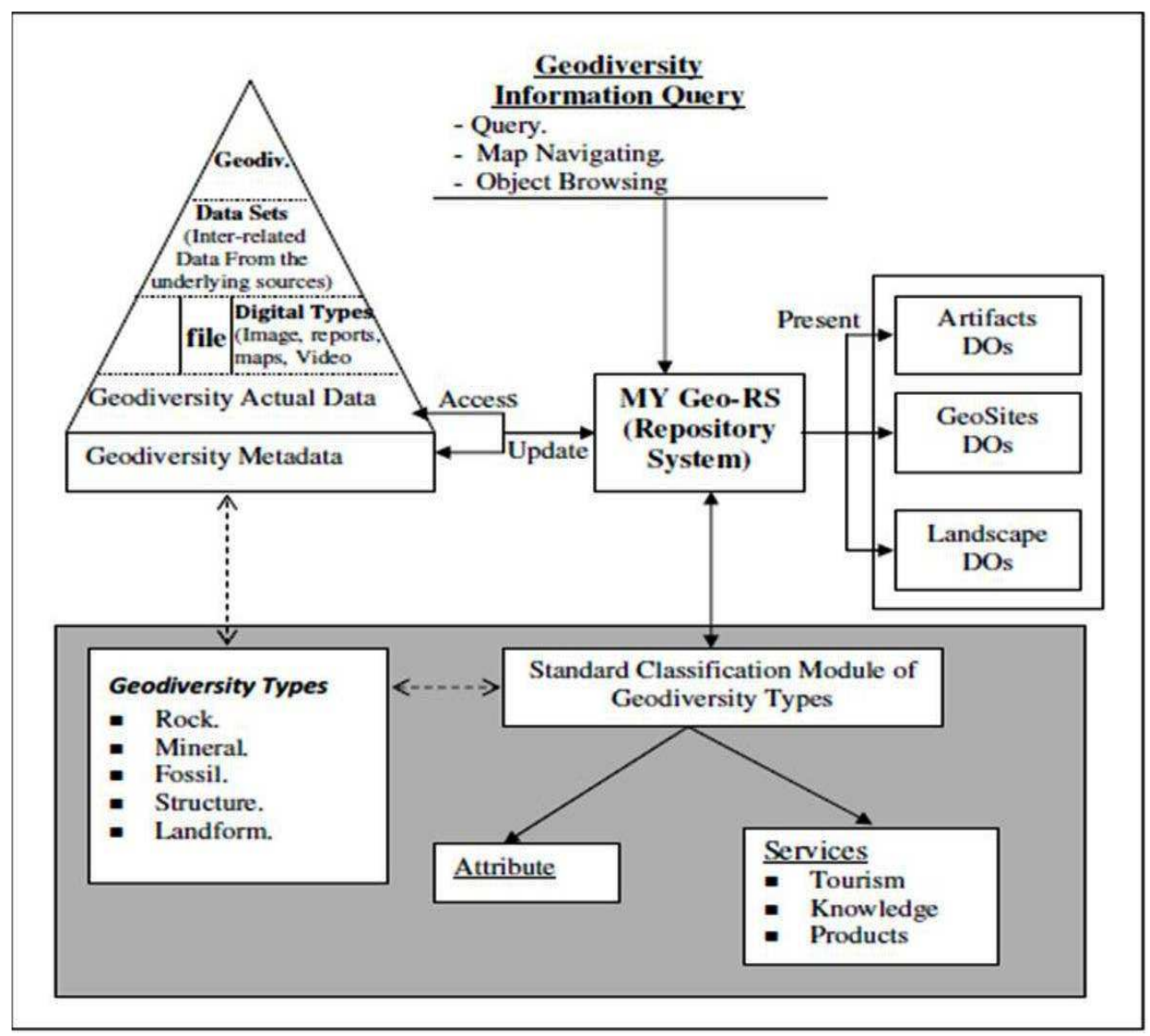

Fig. 1. The Architecture of MyGeo-RS (Aziz, et al., 2009) 


\section{MyGeo-RS : The Design}

There were four steps in the phase of system design in this study. First step was the identification and classification of data requirements and specification. Secondly, was the definition of data structure. The third step was the identification and definition of the system logical structure and finally, was overall system control design.

An issue to be solved in the phase of design was how to realize the structure of the data to be kept in the repository. It was difficult to get a mutual understanding and agreement between the computer experts and the geology experts. The process of identification and classification were very complex and time consuming since it applied several expeditions to the study location. The complexity process is also agreed Paula and Pedro (2004). After series of long and massive discussions and visits, the requirement specifications and structures have been described and documented. At this stage the data requirements have been mapped into several data structures or tables in one database entity (myGeo repository).

Accordingly, MyGeo-RS has thirteen data tables: Landscape, Artifact, Cultural, Fossil, Geopark, Landform, Management, Mineral, Primary_Structure, Recreational, Rock, Secondary_Structure and Reference. Table of Artifact holds data of artifacts collected, table of Cultural maintain data of cultural value, table of Geopark holds data of geosites in Langkawi Island, table of Landform contains data about landform and its associated attributes. Table of Management captures data about management of the geopark. In Langkawi, there are 99 geoparks which consist of thousands of artifacts. Table Mineral maintains data of mineral, table Primary_Structure maintains data under primary structure classification and Secondary_Structure holds data of secondary structure. Table of Recreational stores data of recreational value, table Rock contains information on rocks and Reference table holds data on related references of this project.

\section{Implementation}

The MyGeo-RS was implemented as a database Window system. The functional requirements have been identified and can be summarized as follows: -

a) Capture geodiversity data in different format such as:

- text

- images

- photo

- video/studio

b) Store, update, and delete database content on demand.

c) Display to producing printed output as needed.

d) Import and export database content based on prescribed format.

e) Search for specific content based on "keyword" (full and practical)

MyGeo-RS has four main functions: management module, administration module, operational modules and Internet access module. Management module includes functions of detail description of MyGeo-RS and main page of the system. The administration module provides functions of managing user access control, managing database structure and content, implementing back-up system and managing access-log system. The operation module manipulates multimedia data in this system, which includes functions of input, update, delete, import and export data and reporting. The Internet access modules provide public access based on a simple membership system, query and searching, viewing capabilities based on prescribe format (printer friendly format) and feedback/comment communication.

The system was built using Eclipse and MySql database and runs in Window environment. Figure 2 illustrates the main menu of MyGeo-RS. It shows that there are three categories of users which are site 
administrator, system administrator and general users (which include general public and scientists). This different category of users is to ensure integrity and security of the system and the data. The site administrator is the person who is responsible for handling and maintaining the data while system administrator is the person who is responsible for maintaining the system and users.

Data inputting is implemented at the user side and in this project the user involved is the Geological Heritage Group of Malaysia which is based at Institute for Environment and Development (LESTARI), Universiti Kebangsaan Malaysia. The group is responsible to coordinate and update the data requires by this system. Figure 3 and
Figure 4 shows two snap screens of this system. They illustrate some examples of the list of artifacts in each geoparks and the detail of each artifact with associated image.

Information provided by MyGeo-RS basically reflects the present status of geodiversity information in Langkawi Island, Malaysia. The system is designed that can be applied in other geology parks. In general the system will be beneficial to the Langkawi Research Centre, Geological Heritage Group of Malaysia, tourism industries and general public. As an initial result with initial data provided, MyGeo-RS has been demonstrated to, and received positive comments from the members of Geology Heritage Group of Malaysia.

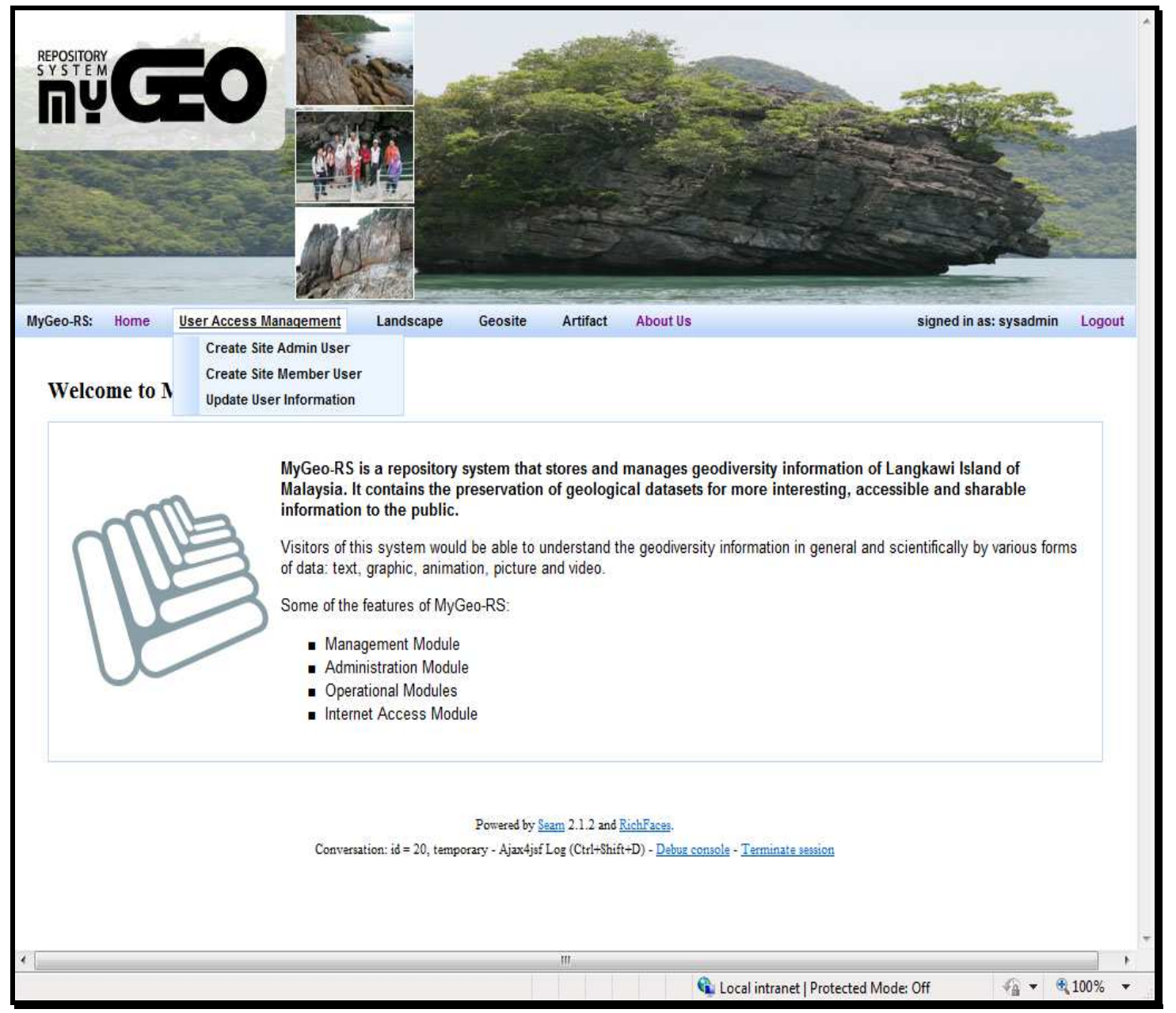

Fig 2: Main Menu of MyGeo-RS 


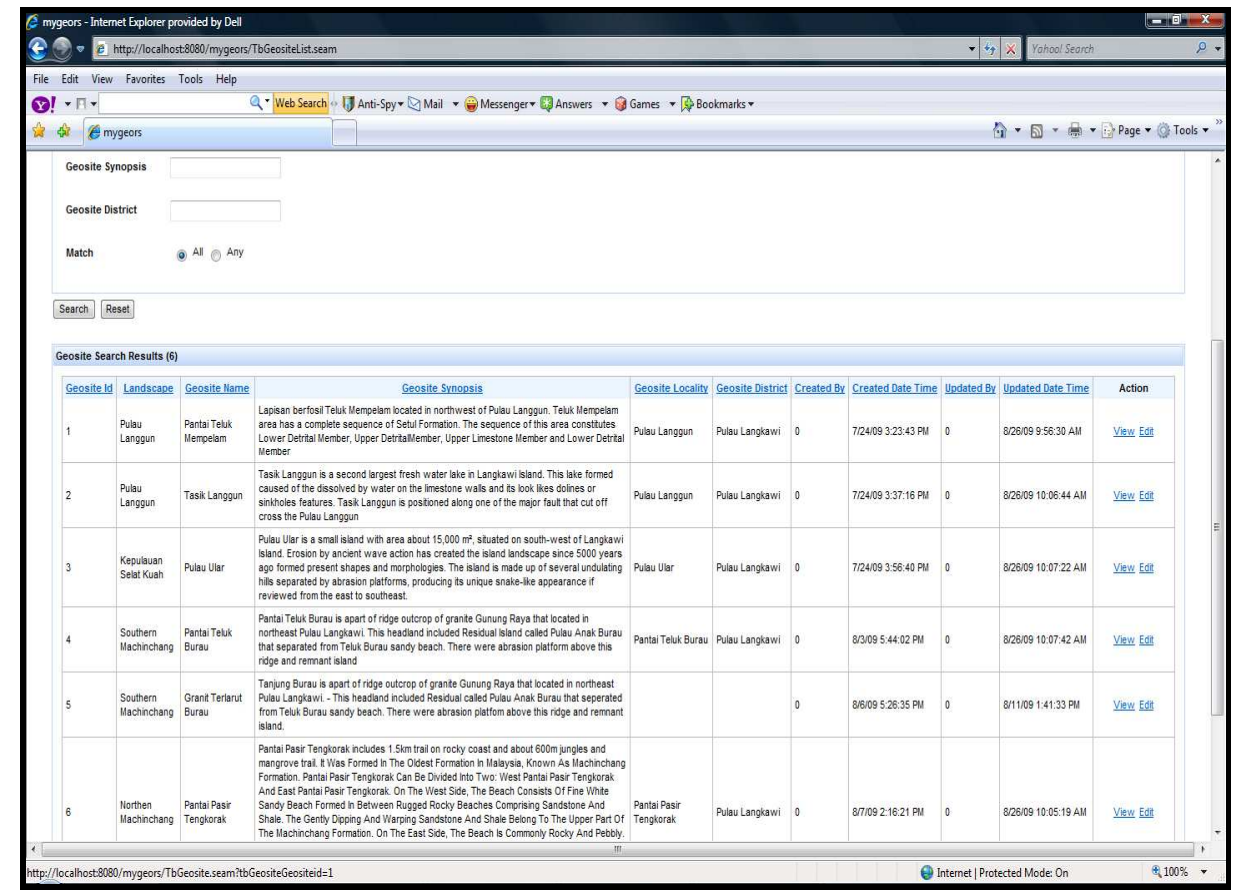

Fig. 3: First Screen Shots of MyGeo-RS (list of Artifacts)

It can be used for general data query, tourism and travel planning for public and tourism agencies as well as geology heritage planning and management. Gaps and priorities in geodiversity conservation can be identified which can support geodiversity planning. The most common uses of the system are dissemination of geodiversity information for the public and data query for scientists.

\section{Discussion and Conclusion}

In Malaysia, information about geodiversity of Langkawi Island is gathered and maintained by several different projects. The information is realized as an important and valuable asset that needed by the scientists, interested agencies and general public. These groups of users must have access to this information easily and efficiently. This means that tools are required to help to locate, analyse and integrate the information in order to improve the quality of decision making and in turn the quality of life (Xu, et al., 1999). 


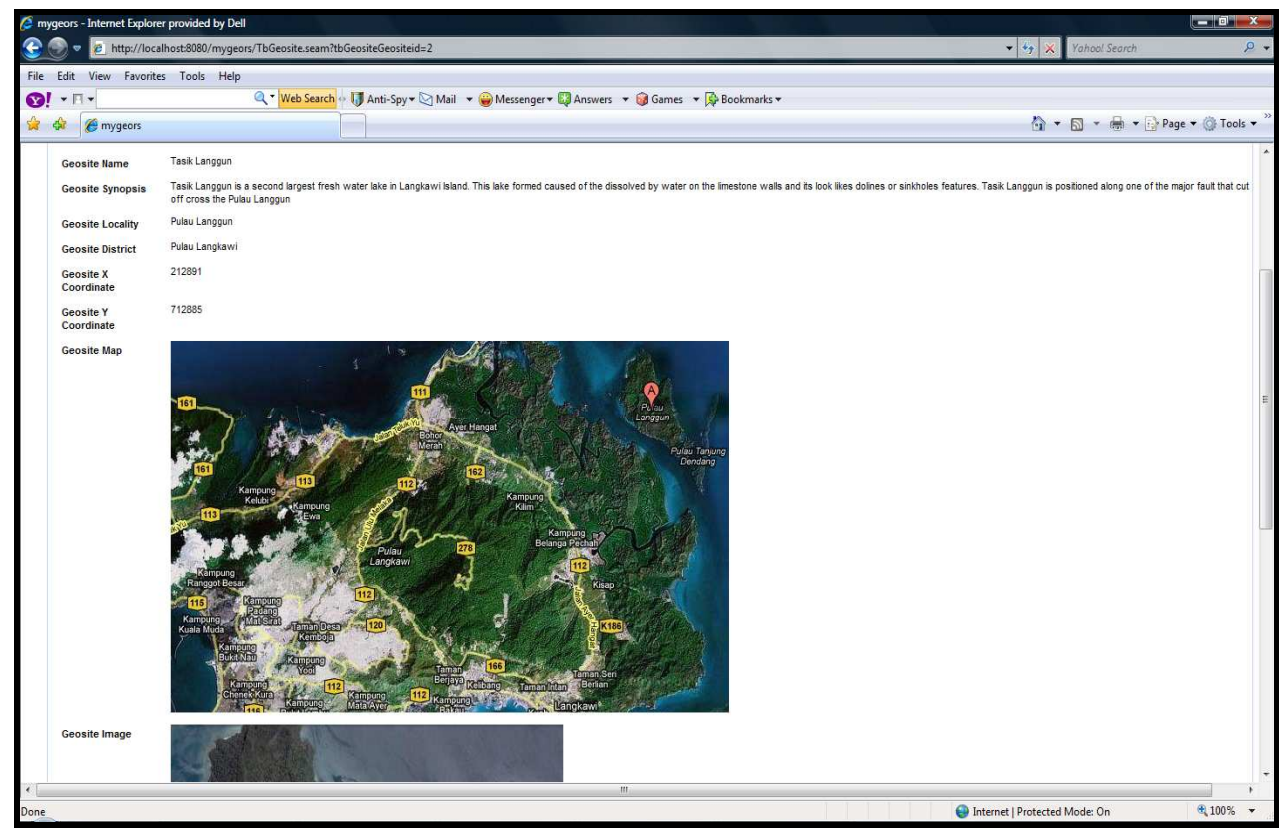

Fig. 4: Second Screen Shots of MyGeo-RS (Artifact's Detail)

The development of MyGeo-RS, a geodiversity repository system is a timely effort and a suitable solution that can assure the management and preservation of geological datasets to be made more interesting, accessible and shared to the public at large. This research was embarked based on the realization that with the advancement of information and communication technology, as well as data mining and knowledge discovery, knowledge about geodiversity can be better represented and explored. This action research attempted to establish on how geodiversity components and their relationships can be modeled into various forms of digital object (DO) to enable access and sharing of geodiversity information. The target user groups of this project are mainly to public users and tourism industry. This means that the data captured and provided in this project is suitable for these groups but at the same time giving little contribution to scientific applications. This will be the limitation of current design and architecture and further work need to be done to accommodate the scientific requirements.

From the experience, the process of developing the standard geology data can be difficult, complex and expensive. Even under these circumstances, however geologists and information technology experts appear willing to compromise to achieve data exchange and standard. Although some standards exist, no coordinated and established comprehensive geodiversity standards been introduced to the community. Some standards must be implemented by the geologists and managers of data. Standards must be kept as flexible as possible to encourage data submission, but should be constructed in such a way that they can be mapped into rigorous standards, and allow interoperable searching. In this research, data quality is not covered. Subsequent efforts are required to perfect the data and standard.

Future work will focus on generating integration with tourism industries and companies as well as scientific applications. As been mentioned earlier, the necessary target of this research was to develop partnership between system managers and the geology related community such as tourism industry and other scientific centers. Therefore, this will be the next phase of this research which targeted to have a linking and partnership with tourism operators and research centers providing the necessary information infrastructure. 


\section{Acknowledgement}

This project was funded by Malaysian Ministry of Science, Technology and Innovation. We also would like to thank you to MSKota Sdn. Bhd. for their effort and cooperation in system development.

\section{References}

Andre, P. Antunes, P. (2004). "SaGISC : A Geo-Collaborative System," Springer-Verlag Berlin Heidelberg, 3, 198.

Aziz, D., Jaradat, A., Sufian, I., Laily, D. \& Niezam, S. (2005). 'Pemodelan Maklumat Biodiversiti: Pendekatan Objek Digital Informatif,' JSChem ITB-UKM VI-2005, 153.

Chang, Y.-S. \& Park, H.-D. (2004). "Development of a Web-based Geographic Information System for the Management of Borehole and Geological Data," Computer and Geosciences, 30(8). 887-897.

Deraman, A., Yahaya, J., Salim, J., Idris, S., Jambari, D. I., Jaradat, A., Komoo, I., Leman, M. S., Unjah, T., Sarman, M. \& Sian, L. C. (2009). "The Development of myGeo-RS: A Knowledge Management System of Geodiversity Data for Tourism Industries," Communication of IBIMA, 8, 142-146 ISSN:1943-7765.

Komoo, I. (2003). 'Conservation Geology Protecting Hidden Treasures of Malaysia,' ASM Inaugural Lectures.

Komoo, I. \& Leman, M. S. (1999). 'Geologi Pemuliharaan Kepulauan Langkawi,' Warisan Geologi Malaysia, 2nd edition : 331.

Lee, S. \& Choi, W. (2001). "Construction of Geological Hazard Spatial DB and Development of Geological Hazard Spatial Information System," Proceedings of the International Geosciences and Remote Sensing Symposium (IGARSS).4, 16931695.

UNESCO. (1988). 'Operational Guideline for the Implementation of the World Heritage Convention. Intergoverment Committee for the Protection of the world cultural and
Natural Heritage,' UNESCO WHC/2/Revised December 1988.

UNESCO. (2008). "United Nation Educational, Scientific and Cultural Organisation," [Online], [Retrieved August 15,2010], http://www.unesco.org/new/en/unesco/

Xu, H., Goa, Z., Xue, D. \& Wu, X. (1999). "China National Biodiversity Information Query System," Journal of Environment Management, 56, 45-59. 